\title{
Integer-empty polytopes in the 0/1-cube with maximal Gomory-Chvátal rank
}

\author{
Sebastian Pokutta ${ }^{\mathrm{a}}$, Andreas S. Schulz ${ }^{\mathrm{b}, *}$ \\ a Friedrich-Alexander-Universität Erlangen-Nürnberg, Germany \\ ${ }^{\mathrm{b}}$ Massachusetts Institute of Technology, USA
}

\section{A R T I C L E I N F O}

\section{Article history:}

Received 6 March 2011

Accepted 13 September 2011

Available online 25 September 2011

\section{Keywords:}

Integer programming

Cutting planes

Gomory-Chvátal closure

Rank

\begin{abstract}
A B S T R A C T
We provide a complete characterization of all polytopes $P \subseteq[0,1]^{n}$ with empty integer hulls, whose Gomory-Chvátal rank is $n$ (and, therefore, maximal). In particular, we show that the first Gomory-Chvátal closure of all these polytopes is identical.
\end{abstract}

(c) 2011 Elsevier B.V. All rights reserved.

\section{Introduction}

The Gomory-Chvátal procedure is a well-known technique to derive valid inequalities for the integral hull $P_{I}$ of a polyhedron $P=\left\{x \in \mathbb{R}^{n} \mid A x \leq b\right\}$. It was introduced by Chvátal [2] and, implicitly, by Gomory [6-8] as a means to establish certain combinatorial properties via cutting-plane proofs. Cutting planes and Gomory-Chvátal cuts, in particular, belong to today's standard toolbox in integer programming. However, despite significant progress in recent years (see, e.g., $[1,3,5,9]$ ), the Gomory-Chvátal procedure is still not fully understood from a theoretical standpoint, especially in the context of polytopes contained in the 0/1-cube. For example, the question whether the currently best known upper bound of $O\left(n^{2} \log n\right)$ on the Gomory-Chvátal rank, established in [5], is tight, remains open. In [5], it was also shown that there is a class of polytopes contained in the $n$-dimensional $0 / 1$-cube whose rank exceeds $n$. (See [11] for a more explicit construction.) However, no family of polytopes in the 0/1-cube is known that realizes super-linear rank, and thus there is a large gap between the best known upper bound and the largest realized rank.

We consider the special case of $P \subseteq[0,1]^{n}$ with $P_{I}=\emptyset$ and Gomory-Chvátal rank $\operatorname{rk}(P)=n$ (i.e., maximal rank, as $\operatorname{rk}(P) \leq n$ holds for all $P \subseteq[0,1]^{n}$ with $P_{I}=\emptyset$; see [1]). This case is of particular interest as, so far, all known proofs of

\footnotetext{
* Correspondence to: Massachusetts Institute of Technology, E62-569, 100 Main Street, Cambridge, MA 02142, USA.

E-mail address: schulz@mit.edu (A.S. Schulz).
}

polynomial upper bounds on the rank of polytopes in the 0/1-cube (cf., $[1,5]$ ) crucially depend on this special case. The improvement from $O\left(n^{3} \log n\right)$ in [1] to $O\left(n^{2} \log n\right)$ in [5] as an upper bound on the rank of polytopes in $[0,1]^{n}$ is a direct consequence of a better upper bound on the rank of certain polytopes in the 0/1-cube that do not contain integral points. It can actually be shown that lower bounds on the rank of polytopes $P \subseteq[0,1]^{n}$ with $P_{I}=\varnothing$ play a crucial role in understanding the rank of any (well-defined) cutting-plane procedure [10]. Moreover, in many cases, the rank of a face $F \subseteq P$ with $F_{I}=\emptyset$ induces a lower bound on the rank of $P$ itself. In fact, the construction of the aforementioned families of polytopes in $[0,1]^{n}$ whose rank is strictly larger than $n$ exploits this connection.

In view of this, a thorough understanding of the GomoryChvátal rank of polytopes $P \subseteq[0,1]^{n}$ with $P_{I}=\emptyset$ might help to derive better upper and lower bounds for the general case. In this paper, we characterize all polytopes $P \subseteq[0,1]^{n}$ with $P_{I}=\emptyset$ and $\operatorname{rk}(P)=n$. In particular, we show that after applying the Gomory-Chvátal procedure once, one always obtains the same polytope. Furthermore, we show that $P \subseteq[0,1]^{n}$ with $P_{I}=\varnothing$ has $\operatorname{rk}(P)=n$ if and only if $P \cap F \neq \emptyset$ for all one-dimensional faces $F$ of the $0 / 1$-cube $[0,1]^{n}$.

The paper is organized as follows. In Section 2, we introduce our notation and recall some basic facts about the Gomory-Chvátal procedure. Afterwards, in Section 3, we derive the characterization of all polytopes $P \subseteq[0,1]^{n}$ with $P_{I}=\emptyset$ and $\operatorname{rk}(P)=n$. In particular, in Section 3.2, we relate the rank of a polytope $P \subseteq[0,1]^{n}$ with $P_{I}=\varnothing$ to the rank of its faces. We then prove the characterization for the two-dimensional case in Section 3.3, which is an essential ingredient for the subsequent generalization to arbitrary dimension in Section 3.4. 


\section{Preliminaries}

Let $P=\left\{x \in \mathbb{R}^{n} \mid A x \leq b\right\}$ be a polytope with $A \in \mathbb{Z}^{m \times n}$ and $b \in \mathbb{Z}^{m}$. The Gomory-Chvátal closure of $P$ is defined as

$P^{\prime}:=\bigcap_{\lambda \in \mathbb{R}_{+}^{m}, \lambda A \in \mathbb{Z}^{n}}\{x: \lambda A x \leq\lfloor\lambda b\rfloor\}$.

The result $P^{\prime}$ is again a polytope (see [2]), and one can apply the operator iteratively. We let $P^{(i+1)}:=\left(P^{(i)}\right)^{\prime}$ for $i \geq 0$ and $P^{(0)}:=P$. The resulting sequence $\left\{P^{(i)}\right\}_{i>0}$ becomes stationary after finitely many steps [2], and the smallest $k$ such that $P^{(k+1)}=P^{(k)}$ is the Gomory-Chvátal rank of $P$ (in the following often rank of $P$ ), denoted by $\operatorname{rk}(P)$. In particular, $P^{(\mathrm{rk}(P))}=P_{I}$, where $P_{I}:=\operatorname{conv}(P \cap$ $\mathbb{Z}^{n}$ ) denotes the integral hull of $P$.

We will make repeated use of the following well-known lemma:

Lemma 2.1 ([4, Lemma 6.33]). Let $P$ be a rational polytope and let $F$ be a face of $P$. Then $F^{\prime}=P^{\prime} \cap F$.

If $P \subseteq[0,1]^{n}$ and $P_{I}=\emptyset$, Lemma 2.1 can be used to derive an upper bound on $\operatorname{rk}(P)$.

Lemma 2.2 ([1, Lemma 3]). Let $P \subseteq[0,1]^{n}$ be a polytope with $P_{I}=\emptyset$. Then $\operatorname{rk}(P) \leq n$.

This bound is actually tight; a family of polytopes $A_{n} \subseteq[0,1]^{n}$ with $\left(A_{n}\right)_{I}=\emptyset$ and $\operatorname{rk}\left(A_{n}\right)=n$ was described in [3, p. 481].

For $i \in[n]$, the $i$-th coordinate flip maps $x_{i} \mapsto 1-x_{i}$ and $x_{j} \mapsto x_{j}$ for $i \neq j$. Another property that we will extensively use is that the Gomory-Chvátal operator is commutative with unimodular transformations, in particular, coordinate flips.

Lemma 2.3 ([5, Lemma 4.3]). Let $P \subseteq[0,1]^{n}$ be a polytope and let $u$ be a coordinate flip. Then $(u(P))^{\prime}=u\left(P^{\prime}\right)$.

Given polytopes $P \subseteq[0,1]^{n}, Q \subseteq[0,1]^{k}$, and a $k$-dimensional face $F$ of $[0,1]^{n}$, we say that $P \cap F \cong Q$ if the canonical projection of $P \cap F$ onto $[0,1]^{k}$ is equal to $Q$. We denote the interior of $P$ by $\operatorname{Int}(P)$ and, with $P, F$, and $Q$ as before, the relative interior of $P$ with respect to $F$ is defined as $\operatorname{RInt}_{F}(P):=\operatorname{Int}(Q)$. We use $e$ to denote the all-one vector, and $\frac{1}{2} e$ to denote the all-one-half vector. If $I \subseteq[n] \times\{0,1\}, \frac{1}{2} e^{I}$ has coordinates $\frac{1}{2} e_{i}^{l}=\frac{1}{2}$ whenever $(i, l) \notin I$, and $\frac{1}{2} e_{i}^{I}=l$ for $(i, l) \in I$. Similarly, if $F$ is a face of $[0,1]^{n}$, we define $\frac{1}{2} e^{F} \in F$ to be $\frac{1}{2}$ in those coordinates not fixed by $F$. Moreover, we define $F_{k}$ to be the set of all vectors $x \in\left\{0, \frac{1}{2}, 1\right\}^{n}$ such that exactly $k$ coordinates are equal to $\frac{1}{2}$, and the remaining coordinates are in $\{0,1\}$. For convenience, we use $[n]:=\{1, \ldots, n\}$ for $n \in \mathbb{N}$.

\section{Polytopes $P \subseteq[0,1]^{n}$ with $P_{I}=\emptyset$ and maximal rank}

For $n \in \mathbb{N}$, we define the polytope $B_{n} \subseteq[0,1]^{n}$ by

$B_{n}:=\left\{x \in[0,1]^{n} \mid \sum_{i \in S} x_{i}+\sum_{i \in[n] \backslash S}\left(1-x_{i}\right) \geq 1\right.$ for all $\left.S \subseteq[n]\right\}$.

Note that, $\left(B_{n}\right)_{I}=\emptyset$. This family of polytopes will be essential to our subsequent discussion.

\subsection{Properties of $B_{n}$}

In the following section, we will characterize $B_{n}^{(k)}$ and show, specifically, that $B_{n}^{(n-2)}=\left\{\frac{1}{2} e\right\}$. Moreover, we will show that $\left\{0, \frac{1}{2}\right\}$-cuts, i.e., Gomory-Chvátal cuts with $\lambda \in\left\{0, \frac{1}{2}\right\}^{m}$, suffice to deduce $\left(B_{n}\right)_{I}=\emptyset$, and the rank with respect to the classical Gomory-Chvátal procedure coincides with the rank if one were to use $\left\{0, \frac{1}{2}\right\}$-cuts only. Clearly, with $B_{n}$ as above and $F$ being a $k$-dimensional face of $[0,1]^{n}$, we have $B_{n} \cap F \cong B_{k}$. As a direct consequence of the proof of [3, Lemma 7.2] one obtains:

Lemma 3.1. Let $P \subseteq[0,1]^{n}$ be a polytope with $F_{k} \subseteq P$ for some $k<n$. Then $F_{k+1} \subseteq \bar{P}^{\prime}$.

Proof. We include a proof for completeness. Let $P$ as above and let $a x<b+1$ with $a \in \mathbb{Z}^{n}$ and $b \in \mathbb{Z}$ be valid for $P$. We have to show that $a p \leq b$ for every $p \in F_{k+1}$. Let $p \in F_{k+1}$ be arbitrary. If $a p \in \mathbb{Z}$, we are done. So assume that $a p \notin \mathbb{Z}$. Then there exists $i \in[n]$ such that $a_{i} \neq 0$ and $p_{i}=\frac{1}{2}$. We define the points $p^{0}, p^{1}$ by setting $p_{j}^{0}=$ $p_{j}^{1}=p_{j}$ for all $j \neq i, p_{i}^{0}=0$, and $p_{i}^{1}=1$. Hence, $p=\frac{1}{2} p^{0}+\frac{1}{2} p^{1}$. Note that, $p^{0}, p^{1} \in F_{k} \subseteq P$ and, therefore, $a p^{l}<b+1$ holds for $l \in\{0,1\}$. We derive $a p+\frac{1}{2} \leq \max \left\{a p^{0}, a p^{1}\right\}<b+1$ and thus $a p<b+\frac{1}{2}$. Since $a p \in \frac{1}{2} \mathbb{Z}$, it follows that $a p \leq b$, hence $p \in P^{\prime}$. As the choice of $p \in F_{k+1}$ was arbitrary, we obtain $F_{k+1} \subseteq P^{\prime}$.

Note that, $F_{2} \subseteq B_{n}$. Thus, by Lemma 3.1, we have:

Corollary 3.2. $F_{k} \subseteq B_{n}^{(k-2)}$.

The following theorem specifies a family of valid inequalities for $B_{n}^{(k)}$.

Theorem 3.3. Let $B_{n}$ be defined as above and $k \leq n$. Then

$\sum_{i \in I} x_{i}+\sum_{i \in \tilde{I} \backslash I}\left(1-x_{i}\right) \geq 1$

is valid for $B_{n}^{(k)}$ for all $I \subseteq \tilde{I} \subseteq[n]$ with $|\tilde{I}|=n-k$. Moreover, these inequalities can be derived as iterated $\left\{0, \frac{1}{2}\right\}$-cuts.

Proof. The proof is by induction on $k$. First, let us look at the case $k=0$. By definition, $\sum_{i \in I} x_{i}+\sum_{i \in \tilde{I} I I}\left(1-x_{i}\right) \geq 1$ with $\tilde{I}=[n]$ is valid for $B_{n}$. Now consider $0<k \leq n$, and assume that the claim holds for $k-1$. Let $\tilde{I} \subseteq[n]$ with $|\tilde{I}|=n-k$ be arbitrary. We have to prove that $\sum_{i \in I} x_{i}+\sum_{i \in \tilde{I} \backslash I}\left(1-x_{i}\right) \geq 1$ with $I \subseteq \tilde{I}$ is valid for $B_{n}^{(k)}$. Let $I_{0}=\tilde{I} \cup\{h\}$ for some $h \notin \tilde{I}$. Note that, such an $h$ exists as $k>0$. Then

$x_{h}+\sum_{i \in I} x_{i}+\sum_{i \in \tilde{I} \backslash I}\left(1-x_{i}\right)=\sum_{i \in I \cup\{h\}} x_{i}+\sum_{i \in I_{0} \backslash(I \cup\{h\})}\left(1-x_{i}\right) \geq 1$

and

$\left(1-x_{h}\right)+\sum_{i \in I} x_{i}+\sum_{i \in \tilde{I} \backslash I}\left(1-x_{i}\right)=\sum_{i \in I} x_{i}+\sum_{i \in I_{0} \backslash I}\left(1-x_{i}\right) \geq 1$

are valid for $B_{n}^{(k-1)}$, by induction hypothesis. By adding the two inequalities, we obtain

$2 \sum_{i \in I} x_{i}+2 \sum_{i \in \tilde{I} \backslash I}\left(1-x_{i}\right) \geq 1$

and, therefore, $\sum_{i \in I} x_{i}+\sum_{i \in \tilde{I} \backslash I}\left(1-x_{i}\right) \geq\left\lceil\frac{1}{2}\right\rceil=1$ is valid for $B_{n}^{(k)}$.

We immediately obtain the following corollary.

Corollary 3.4. $B_{n}^{(n-2)}=\left\{\frac{1}{2} e\right\}$.

Proof. First note that, $\frac{1}{2} e \in B_{n}^{(n-2)}$ by Corollary 3.2. By Theorem 3.3 we know that $\sum_{i \in I} x_{i}+\sum_{i \in \tilde{I} \backslash I}\left(1-x_{i}\right) \geq 1$ with $I \subseteq \tilde{I}=\{u, v\} \subseteq I$ is valid for $B_{n}^{(n-2)}$, for any pair $u, v \in[n], u \neq v$. Therefore $x_{u}+x_{v} \geq$ $1, x_{u}+\left(1-x_{v}\right) \geq 1,\left(1-x_{u}\right)+x_{v} \geq 1$, and $\left(1-x_{u}\right)+\left(1-x_{v}\right) \geq 1$ are valid for $B_{n}^{(n-2)}$, which implies $x_{u}=x_{v}=\frac{1}{2}$. 
The following lemma characterizes the vertices of $B_{n}$.

Lemma 3.5. $B_{n}=\operatorname{conv}\left(F_{2}\right)$.

Proof. Note that, $\operatorname{conv}\left(F_{2}\right) \subseteq B_{n}$. We will show that every vertex $\tilde{x}$ of $B_{n}$ belongs to $F_{2}$, which would complete the proof. So let $\tilde{x}$ be an arbitrary vertex of $B_{n}$.

First, we prove that $\tilde{x}$ is half-integral. Suppose not. Let $D=$ $\left\{i \in[n] \mid \tilde{x}_{i} \notin\left\{0, \frac{1}{2}, 1\right\}\right\}$. By applying appropriate coordinate flips, we may assume, without loss of generality, that $\tilde{x}_{i}<\frac{1}{2}$ for all $i \in D$. Since $\tilde{x}$ is a vertex of $B_{n}$, there exists an index set $I \subseteq$ $[n]$ such that $\sum_{i \in I} \tilde{x}_{i}+\sum_{i \in[n] \backslash I}\left(1-\tilde{x}_{i}\right)=1$. Note that this implies $D \subseteq I$. If there exists $d \in D$ such that $d \notin I$, then $\sum_{i \in I \cup\{d\}} \tilde{x}_{i}+\sum_{i \in[n] \backslash(I \cup\{d\})}\left(1-\tilde{x}_{i}\right)<1-$ a contradiction. We also obtain $|D|>1$; otherwise the inequality cannot hold at equality. Let $s_{I}=\sum_{i \in I} \tilde{x}_{i}+\sum_{i \in[n] \backslash I}\left(1-\tilde{x}_{i}\right)-1$ for all $I \subseteq[n]$. As $|D| \geq 2$, there exists $I \subseteq[n]$ with $s_{I}>0$. (Just choose any $I$ with $I \cap D=\emptyset$ ). Let $s=\min _{I \subseteq[n], s_{I}>0} s_{I}$, and let $j, k \in D, j \neq k$. For some sufficiently small $0<\delta<\frac{1}{2} s$, we define $y, z \in[0,1]^{n}$ with $y_{i}=\tilde{x}_{i}=z_{i}$ for all $j \neq i \neq k$ and $y_{j}=\tilde{x}_{j}+\delta, y_{k}=\tilde{x}_{k}-\delta, z_{j}=\tilde{x}_{j}-\delta$, and $z_{k}=\tilde{x}_{k}+\delta$. Note that $\tilde{x}=\frac{1}{2}(y+z)$. It remains to show that $y, z \in B_{n}$, which would contradict that $\tilde{x}$ is a vertex of $B_{n}$. We have earlier seen that whenever $\sum_{i \in I} \tilde{x}_{i}+\sum_{i \in[n] \backslash I}\left(1-\tilde{x}_{i}\right)=1$ holds for some $I \subseteq[n]$, then $D \subseteq I$. Therefore, $\sum_{i \in I} y_{i}+\sum_{i \in[n] \backslash I}\left(1-y_{i}\right)=$ $\sum_{i \in I} \tilde{x}_{i}+\sum_{i \in[n] \backslash I}\left(1-\tilde{x}_{i}\right)+\delta-\delta=1$ as $D \subseteq I$. Moreover, whenever $\sum_{i \in I} \tilde{x}_{i}+\sum_{i \in[n] \backslash I}\left(1-\tilde{x}_{i}\right)>1$ holds for $I \subseteq[n]$, then

$$
\begin{aligned}
\sum_{i \in I} y_{i}+\sum_{i \in[n] \backslash I}\left(1-y_{i}\right) & \geq \sum_{i \in I} \tilde{x}_{i}+\sum_{i \in[n] \backslash I}\left(1-\tilde{x}_{i}\right)-2 \delta \\
& \geq \sum_{i \in I} \tilde{x}_{i}+\sum_{i \in[n] \backslash I}\left(1-\tilde{x}_{i}\right)-s \geq 1 .
\end{aligned}
$$

Thus, $y \in B_{n}$, and $z \in B_{n}$ follows similarly. Consequently, $\tilde{x}$ is halfintegral.

To finish the proof, we show that $\tilde{x}$ has exactly two coordinates that are equal to $\frac{1}{2}$. Suppose that there are more than two entries equal to $\frac{1}{2}$. Then $\sum_{i \in I} \tilde{x}_{i}+\sum_{i \in[n] \backslash I}\left(1-\tilde{x}_{i}\right) \geq \frac{3}{2}$ for all $I \subseteq[n]$. Similarly, less than two entries equal to $\frac{1}{2}$ is not possible as we would obtain $\sum_{i \in I} \tilde{x}_{i}+\sum_{i \in[n] \backslash I}\left(1-\tilde{x}_{i}\right)=\frac{1}{2}<1$ for $I=\{i \in$ $\left.[n] \mid \tilde{x}_{i}=0\right\}$. Hence, $\tilde{x} \in F_{2}$.

We conclude this section by relating $B_{n}$ to arbitrary polytopes $P \subseteq[0,1]^{n}$ with $P_{I}=\emptyset$.

Theorem 3.6. Let $P \subseteq[0,1]^{n}$ with $P_{I}=\emptyset$. Then $P^{(l)} \subseteq B_{n}^{(l-1)}$.

Proof. Let $p \in\{0,1\}^{n}$ be arbitrary, and let $I:=\left\{i \in[n] \mid p_{i}=0\right\}$. As $P_{I}=\emptyset$, we can find $\epsilon_{p}>0$ such that $\sum_{i \in I} x_{i}+\sum_{i \in[n] \backslash I}(1-$ $\left.x_{i}\right) \geq \epsilon_{p}$ is valid for $P$, whereas $\sum_{i \in I} p_{i}+\sum_{i \in[n] \backslash I}\left(1-p_{i}\right)=0$; the inequality separates $p$ from $P$. In particular, we know that $\sum_{i \in I} x_{i}+\sum_{i \in[n] \backslash I}\left(1-x_{i}\right) \geq 1$ is valid for $P^{\prime}$. Since $p \in\{0,1\}^{n}$ was chosen arbitrarily, we obtain that $\sum_{i \in I} x_{i}+\sum_{i \in[n] \backslash I}\left(1-x_{i}\right) \geq 1$ is valid for $P^{\prime}$ for every $I \subseteq[n]$, which implies $P^{\prime} \subseteq B_{n}$. The claim follows from the fact that the Gomory-Chvátal procedure maintains inclusions.

\subsection{The sandwich theorem}

In this section, we will derive bounds on the growth of the rank of a polytope $P \subseteq[0,1]^{n}$ with $P_{I}=\emptyset$.

Theorem 3.7 (Sandwich Theorem). Let $P \subseteq[0,1]^{n}$ with $P_{I}=\emptyset$. Then

$k \leq \operatorname{rk}(P) \leq k+1$

where $k=\max _{(i, l) \in[n] \times\{0,1\}} \operatorname{rk}\left(P \cap\left\{x_{i}=l\right\}\right)$. Moreover, if there exist $i \in[n]$ and $l \in\{0,1\}$ such that $\operatorname{rk}\left(P \cap\left\{x_{i}=l\right\}\right)<k$, then $\operatorname{rk}(P)=k$.
Proof. Clearly, $k \leq \operatorname{rk}(P)$ as there exists $(i, l) \in[n] \times\{0,1\}$ such that $\operatorname{rk}\left(P \cap\left\{x_{i}=l\right\}\right)=k$. For the other inequality, observe that $P^{(k)} \cap\left\{x_{i}=l\right\}=\left(P \cap\left\{x_{i}=l\right\}\right)^{(k)}=\emptyset$, by Lemma 2.1. It follows that $x_{i}<1$ and $x_{i}>0$ are valid for $P^{(k)}$ for all $i \in[n]$. Hence $x_{i} \leq 0$ and $x_{i} \geq 1$ are valid for $P^{(k+1)}$ for all $i \in[n]$, and, therefore, $P^{(k+1)}=\emptyset$, i.e., $\operatorname{rk}(P) \leq k+1$.

It remains to show that $\operatorname{rk}(P)=k$ if there exist $i \in[n]$ and $l \in\{0,1\}$ such that $m:=\operatorname{rk}\left(P \cap\left\{x_{i}=l\right\}\right)<k$. Without loss of generality, we may assume that $l=1$; otherwise we can apply the corresponding coordinate flip. Then $P^{(m)} \cap\left\{x_{i}=l\right\}=\emptyset$ and thus $x_{i}<1$ is valid for $P^{(m)}$. Hence, $x_{i} \leq 0$ is valid for $P^{(k)}$. It follows that $P^{(k)}=P^{(k)} \cap\left\{x_{i}=0\right\}=\left(P \cap\left\{x_{i}=0\right\}\right)^{(k)}=\emptyset$, which implies $\operatorname{rk}(P) \leq k$.

The upper bound in Theorem 3.7 is tight, as can be seen by considering the polytope $A_{n}$, introduced in [3, p. 481], whose definition is identical to that of $B_{n}$ except for the right-hand side, which is $\frac{1}{2}$. Then $\operatorname{rk}\left(A_{n}\right)=n$ and $A_{n}$ satisfies the assumptions of the theorem. As $A_{n} \cap\left\{x_{i}=l\right\} \cong A_{n-1}$, we obtain that $\operatorname{rk}\left(A_{n} \cap\left\{x_{i}=\right.\right.$ $l\})=n-1$ for all $i \in[n]$ and $l \in\{0,1\}$.

However, it is important to note that $\operatorname{rk}\left(P \cap\left\{x_{i}=l\right\}\right)=k$ for all $(i, l) \in[n] \times\{0,1\}$ is not sufficient for $\operatorname{rk}(P)=k+1$. By induction, we immediately obtain a necessary condition for $\operatorname{rk}(P)=n$.

Corollary 3.8. Let $P \subseteq[0,1]^{n}$ be a polytope with $P_{I}=\emptyset$ and $\operatorname{rk}(P)=n$. Then

$\operatorname{rk}(P \cap F)=k$

for all $k$-dimensional faces $F$ of $[0,1]^{n}, 1 \leq k \leq n$.

For the special case of $k=1$, Corollary 3.8 was known before [5, Proof of Proposition 2.4].

\subsection{The two-dimensional case}

In this section, we will provide a full characterization of polytopes $P \subseteq[0,1]^{2}$ with $P_{I}=\emptyset$ and $\operatorname{rk}(P)=2$. We will prove that $P \subseteq[0,1]^{2}$ with $P_{I}=\emptyset$ has rank 2 if and only if $P \cap\left\{x_{i}=l\right\} \neq \varnothing$ for all $(i, l) \in[2] \times\{0,1\}$, which happens if and only if $\frac{1}{2} e \in P^{\prime}$. In case $P$ is a half-integral polytope, the latter condition is equivalent to $\frac{1}{2} e \in \operatorname{Int}(P)$. The following theorem establishes the first part.

Theorem 3.9. Let $P \subseteq[0,1]^{2}$ be a polytope with $P_{I}=\emptyset$. Then $P \cap\left\{x_{i}=l\right\} \neq \emptyset$ for all $(i, l) \in[2] \times\{0,1\}$ if and only if $\operatorname{rk}(P)=2$.

Proof. First, we assume that $P$ contains points $x^{0}=\left(c_{0}, 0\right), x^{1}=$ $\left(0, c_{1}\right), x^{2}=\left(c_{2}, 1\right)$, and $x^{3}=\left(1, c_{3}\right)$. As the rank is monotone, we may assume that these are the only intersections of $P$ with the boundary of the unit cube. Note that, $c_{i} \in(0,1)$ for $0 \leq i \leq 3$. Let $a x<b+1$ with $a \in \mathbb{Z}^{2}$ and $b \in \mathbb{Z}$ be valid for $P$. It is sufficient to prove that $a\left(\frac{1}{2} e\right) \leq b$ as this implies that $\frac{1}{2} e \in P^{\prime} \neq \varnothing$. By using coordinate flips if necessary, we may assume that $a \geq 0$. Consequently, either $x^{2}$ or $x^{3}$ is maximizing a over $P$. We claim that $a x^{m}-a\left(\frac{1}{2} e\right) \geq \frac{1}{2}$ for some $m \in\{2,3\}$. This is sufficient to prove our hypothesis as $a\left(\frac{1}{2} e\right) \leq a x^{m}-\frac{1}{2}<b+1-\frac{1}{2}=b+\frac{1}{2}$ and as $a\left(\frac{1}{2} e\right) \in \frac{1}{2} \mathbb{Z}$, we obtain $a\left(\frac{1}{2} e\right) \leq b$. We distinguish three cases.

Case $a_{2}=a_{1}$. We obtain that $a\left(\frac{1}{2} e\right) \in \mathbb{Z}$ and, therefore, $a\left(\frac{1}{2} e\right) \leq b$.

Case $a_{2} \geq a_{1}+1$. It suffices to show that

$$
\begin{aligned}
a x^{2}-a\left(\frac{1}{2} e\right) \geq \frac{1}{2} & \Leftrightarrow a_{1} c_{2}+a_{2}-\frac{1}{2} a_{1}-\frac{1}{2} a_{2} \geq \frac{1}{2} \\
& \Leftrightarrow\left(c_{2}-\frac{1}{2}\right) a_{1}+\frac{1}{2} a_{2} \geq \frac{1}{2} .
\end{aligned}
$$


This is true because $\left(c_{2}-\frac{1}{2}\right) a_{1}+\frac{1}{2} a_{2} \geq\left(c_{2}-\frac{1}{2}\right) a_{1}+\frac{1}{2}\left(a_{1}+1\right)=$ $c_{2} a_{1}-\frac{1}{2} a_{1}+\frac{1}{2} a_{1}+\frac{1}{2}=c_{2} a_{1}+\frac{1}{2} \geq \frac{1}{2}$.

Case $a_{1} \geq a_{2}+1$. It suffices to show that $a x^{3}-a\left(\frac{1}{2} e\right) \geq \frac{1}{2}$, which follows similarly.

For the other direction, observe that if there exists $(i, l) \in[2] \times$ $\{0,1\}$ such that $P \cap\left\{x_{i}=l\right\}=\emptyset$, then $\operatorname{rk}(P) \leq 1$ follows with Corollary 3.8 .

The following theorem is our main result for the twodimensional case:

Theorem 3.10. Let $P \subseteq[0,1]^{2}$ be a polytope with $P_{I}=\emptyset$. Then the following are equivalent:

(a) $\operatorname{rk}(P)=2$;

(b) $P \cap\left\{x_{i}=l\right\} \neq \varnothing$ for all $(i, l) \in[2] \times\{0,1\}$;

(c) $P^{\prime}=\left\{\frac{1}{2} e\right\}$.

Proof. By Theorem 3.9, (a) $\Leftrightarrow$ (b). Clearly, if $P^{\prime}=\left\{\frac{1}{2} e\right\}$, then $\operatorname{rk}(P)=2$. For the other direction, observe that, by Theorem 3.6, $P^{\prime} \subseteq B_{2}=\left\{\frac{1}{2} e\right\}$ and thus, if $\operatorname{rk}(P)=2$, it follows that $P^{\prime}=$ $\left\{\frac{1}{2} e\right\}$.

We conclude this section with the following lemma showing that whenever $\operatorname{rk}(P)=2$, then $\frac{1}{2} e \in \operatorname{Int}(P)$.

Lemma 3.11. If $P \subseteq[0,1]^{2}$ is a polytope with $P_{I}=\emptyset$ and $\frac{1}{2} e \notin$ $\operatorname{Int}(P)$, then there exists $(i, l) \in[2] \times\{0,1\}$ such that $P \cap\left\{x_{i}=l\right\}=\emptyset$. In particular, if $\operatorname{rk}(P)=2$ then $\frac{1}{2} e \in \operatorname{Int}(P)$.

Proof. The proof of the first part is by contradiction. So let $P \subseteq$ $[0,1]^{2}$ be a polytope with $P_{I}=\emptyset$ and $\frac{1}{2} e \notin \operatorname{Int}(P)$. Suppose $P \cap\left\{x_{i}=l\right\} \neq \varnothing$ for all $(i, l) \in[2] \times\{0,1\}$. Then there exists $\tilde{x} \in P \cap\left\{x_{\tilde{i}}=\tilde{l}\right\}$ with $(\tilde{i}, \tilde{l}) \in[2] \times\{0,1\}$ and $a \in \mathbb{R}^{2}$ such that $a x \leq a\left(\frac{1}{2} e\right)$ is valid for $P$ and $a \tilde{x}=a\left(\frac{1}{2} e\right)$ (i.e., $a x=a\left(\frac{1}{2} e\right)$ is the hyperplane defined by the points $\tilde{x}$ and $\frac{1}{2} e$ ). Without loss of generality, we may assume that $\tilde{i}=1$ and $\tilde{l}=0$; otherwise we can apply coordinate permutations and flips. Then $\tilde{x}$ is of the form $\tilde{x}=(0, c)$ with $c \in(0,1)$, as $P_{I}=\varnothing$. It is easy to see that the hyperplanes $a x=a\left(\frac{1}{2} e\right)$ and $x_{1}=1$ intersect in the point $\tilde{y}=(1,1-c)$. Note that, $\tilde{y}$ is not necessarily in $P$. Let $Q=$ $[0,1]^{2} \cap\left\{a x \leq a\left(\frac{1}{2} e\right)\right\}$, and note that $P \subseteq Q$. If we maximize $x_{2}$ over $P$, we get $\max _{x \in P} x_{2} \leq \max _{x \in Q} x_{2}=\max _{x \in\{(1,1-c),(0, c)\}} x_{2}<1$, contradicting our assumption that $P \cap\left\{x_{i}=l\right\} \neq \emptyset$ for all $(i, l) \in$ $[2] \times\{0,1\}$. The second claim follows from Theorem 3.10.

Clearly, whenever $P$ is half-integral, then $\frac{1}{2} e \in \operatorname{Int}(P)$ if and only if $P \cap\left\{x_{i}=l\right\} \neq \emptyset$ for all $(i, l) \in[2] \times\{0,1\}$. In this case, we therefore obtain $\frac{1}{2} e \in \operatorname{Int}(P)$ if and only if $\operatorname{rk}(P)=2$. If $P$ is not half-integral, however, then this may not be true. Namely, consider $P$ with $\left|P \cap\left\{x_{i}=l\right\}\right|=1$ for all $(i, l) \in[2] \times\{0,1\}$, and move the vertex of the form $(p, 1)$ inwards to $(p, 1-\epsilon)$, for some $\epsilon>0$. It is easy to see that $\epsilon$ can be chosen such that $\frac{1}{2} e$ remains in the interior, however the rank of the resulting polytope is 1 .

\subsection{The general case}

In this section, we provide a complete characterization of all polytopes $P \subseteq[0,1]^{n}$ with $P_{I}=\emptyset$ and $\operatorname{rk}(P)=n$. The following is the main theorem of this paper.
Theorem 3.12. Let $P \subseteq[0,1]^{n}$ be a polytope with $P_{I}=\emptyset$. Then the following statements are equivalent:

(a) $\operatorname{rk}(P)=n$;

(b) $P^{\prime}=B_{n}$;

(c) $F \cap P \neq \varnothing$ for all one-dimensional faces $F$ of $[0,1]^{n}$;

(d) $\operatorname{rk}(P \cap F)=k$ for all $k$-dimensional faces $F$ of $[0,1]^{n}$.

Proof. First, we show that (c) implies (b). So let us assume that $H \cap P \neq \varnothing$ for all one-dimensional faces $H$ of $[0,1]^{n}$. Consider $Q=P \cap F$ for some arbitrary two-dimensional face $F$ of $[0,1]^{n}$. Then $F=\bigcap_{(i, l) \in I}\left\{x_{i}=l\right\}$ for some $I \subseteq[n] \times\{0,1\}$ with $|I|=n-2$. Let $J=[n] \backslash I$. Then $Q \cap\left\{x_{i}=l\right\} \neq \varnothing$ for all $(i, l) \in J \times\{0,1\}$ as $F \cap\left\{x_{i}=l\right\}$ is a one-dimensional face of $[0,1]^{n}$. Theorem 3.10 implies that $\tilde{Q}^{\prime}=\left\{\frac{1}{2} e\right\}$, where $\tilde{Q} \cong Q$ and $\tilde{Q} \subseteq[0,1]^{2}$. Thus, $Q^{\prime}=\left\{\frac{1}{2} e^{I}\right\}$. As the choice of $I$ was arbitrary, we get $F_{2} \subseteq P^{\prime}$. By Lemma 3.5, $B_{n} \subseteq P^{\prime}$ follows. Theorem 3.6 yields $P^{\prime} \subseteq B_{n}$, which completes the proof of (b).

Now assume that $P^{\prime}=B_{n}$. Corollary 3.4 gives $\left\{\frac{1}{2} e\right\}=B_{n}^{(n-2)}=$ $P^{(n-1)}$. Together with Lemma 2.2 , we obtain that $\operatorname{rk}(P)=n$. So $(\mathrm{b})$ implies (a).

By Corollary 3.8, $\operatorname{rk}(P)=n$ implies $F \cap P \neq \varnothing$ for all $k$ dimensional faces $F$ of $[0,1]^{n}$. That is, (d) follows from (a).

The missing implication, (d) to (c), is trivial.

It is a direct consequence of Theorem 3.12 that, for any $n \in \mathbb{N}$, the only half-integral polytope $P \subseteq[0,1]^{n}$ with maximal rank and $P_{I}=\varnothing$ is $A_{n}$. Theorem 3.12 also implies that optimizing a linear function $c$ over $P^{\prime}$ can be done in polynomial time for polytopes $P \subseteq[0,1]^{n}$ with $P_{I}=\emptyset$ and $\operatorname{rk}(P)=n$. It suffices to apply coordinate flips so that $c \geq 0$, to then permute the coordinates such that $c_{1} \geq c_{2} \geq \cdots \geq c_{n}$, and to finally choose the optimal vertex from $F_{2}$.

\section{Acknowledgments}

The authors are grateful to the reviewer for his constructive comments, which really helped to improve the presentation of this paper.

\section{References}

[1] A. Bockmayr, F. Eisenbrand, M. Hartmann, A.S. Schulz, On the Chvátal rank of polytopes in the 0/1 cube, Discrete Applied Mathematics 98 (1999) 21-27.

[2] V. Chvátal, Edmonds polytopes and a hierarchy of combinatorial problems, Discrete Mathematics 4 (1973) 305-337.

[3] V. Chvátal, W. Cook, M. Hartmann, On cutting-plane proofs in combinatorial optimization, Linear Algebra and its Applications 114 (1989) 455-499.

[4] W. Cook, W.H. Cunningham, W.R. Pulleyblank, A. Schrijver, Combinatorial Optimization, John Wiley, 1998.

[5] F. Eisenbrand, A.S. Schulz, Bounds on the Chvátal rank of polytopes in the 0/1cube, Combinatorica 23 (2003) 245-261.

[6] R.E. Gomory, Outline of an algorithm for integer solutions to linear programs, Bulletin of the American Mathematical Society 64 (1958) 275-278.

[7] R.E. Gomory, Solving linear programming problems in integers, in: Proceedings of Symposia in Applied Mathematics X, American Mathematical Society, 1960, pp. 211-215.

[8] R.E. Gomory, An algorithm for integer solutions to linear programs, in: Recent Advances in Mathematical Programming, McGraw-Hill, 1963, pp. 269-302.

[9] M. Hartmann, M. Queyranne, Y. Wang, On the Chvátal rank of certain inequalities, Lecture Notes in Computer Science 1610 (1999) 218-233.

[10] S. Pokutta, A.S. Schulz, On the rank of cutting-plane proof systems, Lecture Notes in Computer Science 6080 (2010) 450-463.

[11] S. Pokutta, G. Stauffer, Lower bounds for the Chvátal-Gomory closure in the 0/1 cube, Operations Research Letters 39 (2011) 200-203. 